\title{
El páramo andino: características territoriales y estado ambiental. Aportes interdisciplinarios para su conocimiento ${ }^{1}$
}

\section{The Andean Páramo: geographic characterization and state of their environment. An interdisciplinary contribution.}

\author{
David Serrano Giné* y Remigio Galárraga Sánchez**
}

\section{INTRODUCCIÓN}

En las latitudes intertropicales del planeta, en los cuatro continentes cruzados por la línea ecuatorial, se encuentran elevaciones montañosas con alturas por encima del límite superior del bosque. Estos ambientes, de carácter frío y húmedo, cubiertos por formaciones arbustivas y herbáceas, se designan de forma genérica con el nombre de peatland, es decir, turberas y de forma más específica como «orobioma de estrato suprasilvatico de montañas tropicales con clima de oscilación diaria», según la nomenclatura clásica de Walter (1998).

En África su localización se da en cimas y altiplanicies aisladas de Guinea Ecuatorial, Camerún, Lesoto, Kenia y Etiopía, mientras que en Asia y Oceanía

${ }^{1}$ Esta colaboración ha podido realizarse gracias al programa Prometeo, una iniciativa del gobierno del Ecuador que pretende fortalecer las instituciones de educación superior de este país.

* David Serrano Giné, Departamento de Geografía, Universidad Rovira i Virgili, c/ Joanot Martorell, 15, 43480 Vila-seca (Tarragona) España.

** Remigio Galárraga Sánchez. Departamento de Ingeniería Civil y Ambiental, Escuela Politécnica Nacional. c/ Ladrón de Guevara, E11-253, La Floresta, Quito, Ecuador. 
FIGURA 1

LOCALIZACIÓN DEL PÁRAMO ANDINO

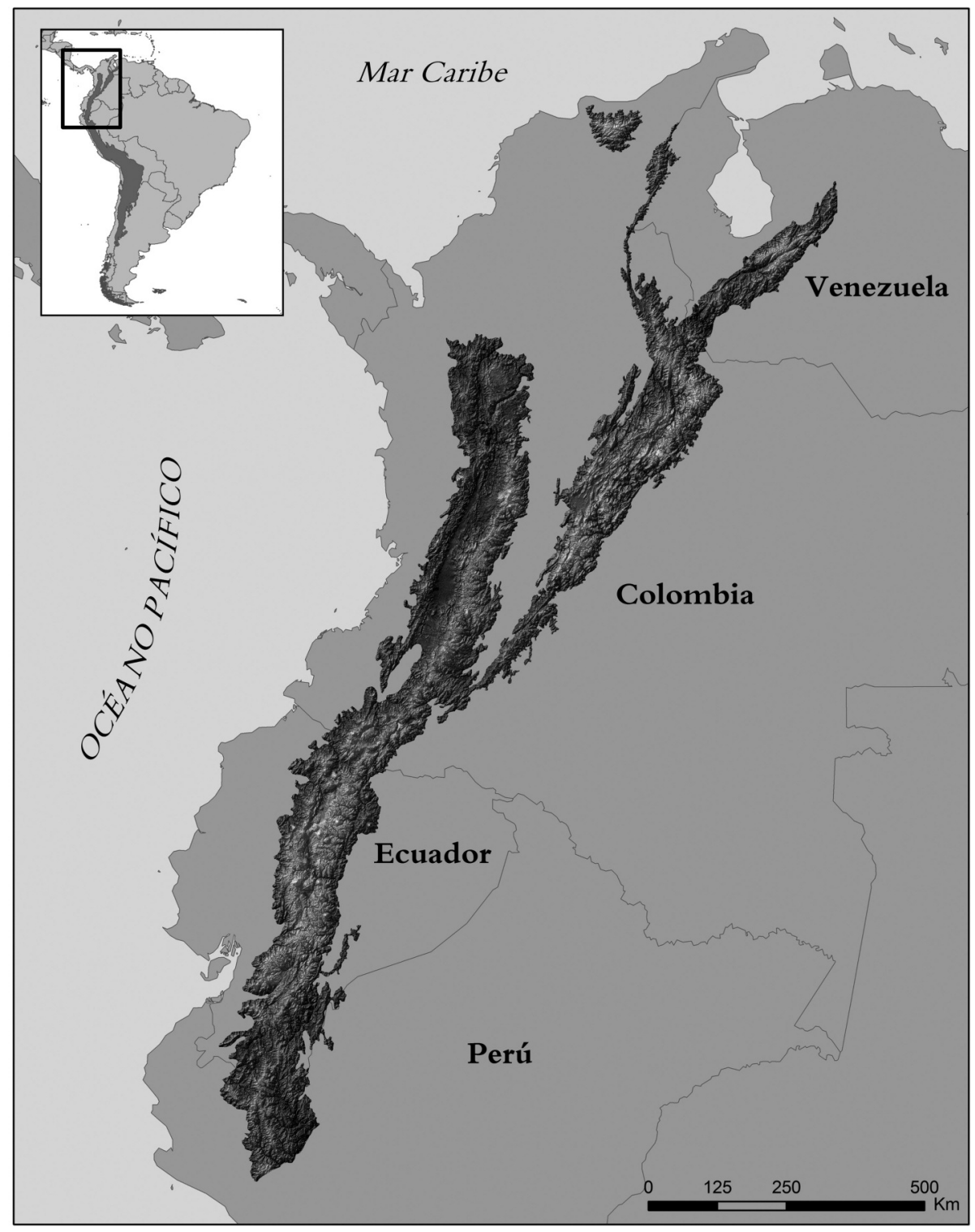

Fuente: elaboración propia.

Estudios Geográficos, Vol. LXXVI, 278, pp. 369-393, enero-junio 2015

ISSN: 0014-1496, eISSN: 1988-8546, doi: 10.3989/estgeogr.201513 
se distribuye en montes isolados de Taiwán, Indonesia y Nueva Guinea. En América su distribución es mucho más pródiga; se distingue un núcleo ístmico y otro andino. Exceptuado las elevaciones de la cordillera de Talamanca (Costa Rica) y su prolongación panameña por la cordillera Central, la continuidad de este ecosistema se extiende por todos los Andes tropicales, desde la cordillera de Mérida (Venezuela) hasta la depresión de Huancabamba (Perú), pasando por la Sierra Nevada de Santa Marta (Colombia) y el valle interandino de Ecuador. Más allá de Huancabamba, y tras una franja de transición denominada jalca, la extensión de los Andes hacia el sur se dilata por Perú y Bolivia en un continuo de altiplanos de carácter árido agrupados bajo el genérico de puna (figura 1). Precisamente esta combinación de alturas elevadas y balance hídrico positivo permite diferenciar el páramo andino de otros ecosistemas circundantes (Ange, 2002; Castaño, 2002; Hofstede, Segarra y Mena, 2003).

La definición arquetípica de Cuatrecasas (1958: 221) define el páramo como:

extensas regiones desarboladas que coronan las sumidas de las cordilleras por encima del bosque andino, desde $3800 \mathrm{~m}$ s.n.m (localmente $3200 \mathrm{~m}$ s.n.m.) y que pueden subdividirse en los subpisos: subpáramo, páramo propiamente dicho y superpáramo (figura 2).

Otras definiciones centran su atención en aspectos de orden abiótico, como el relieve, las aguas y las condiciones climáticas (De Bièvre, Íñiguez y Buytaert, 2006), o en la comunión de elementos bióticos y abióticos, dando lugar a caracterizaciones de orden ecológico, donde toma especial importancia el relieve periglaciar, la oscilación diaria de temperatura y las adaptaciones de la fauna y la flora (Guhl, 1982; Van der Hammen, 1997). Aún existen otras aportaciones centradas en aspectos sociales y económicos, que consideran el páramo como un espacio eminentemente cultural con una gran carga social y antropológica (Ramón, 2002; Girard, 2005; Varela, 2008).

Definir el páramo no resulta sencillo, pues en él convergen particularidades de carácter climático, geomorfológico, biogeográfico y cultural (Medina y Mena, 2001). El páramo, pues, no es únicamente un ecosistema de la alta montaña tropical, sino también un espacio de producción y trabajo con una gran carga histórica, cultural y política, es decir, un paisaje, en la mayor complejidad del término. Esta ambigüedad terminológica dificulta su caracterización y, más aún, una delimitación homogénea. Así, las dimensiones del páramo se estiman en poco más de $46.000 \mathrm{~km}^{2}$, distribuidos mayormente en Ecuador $(39,8 \%)$, Colombia $(30,5 \%)$ y Perú $(24,6 \%)$ y, en menor medida, Ve- 
FIGURA 2

ASPECTO DEL PÁRAMO ANDINO

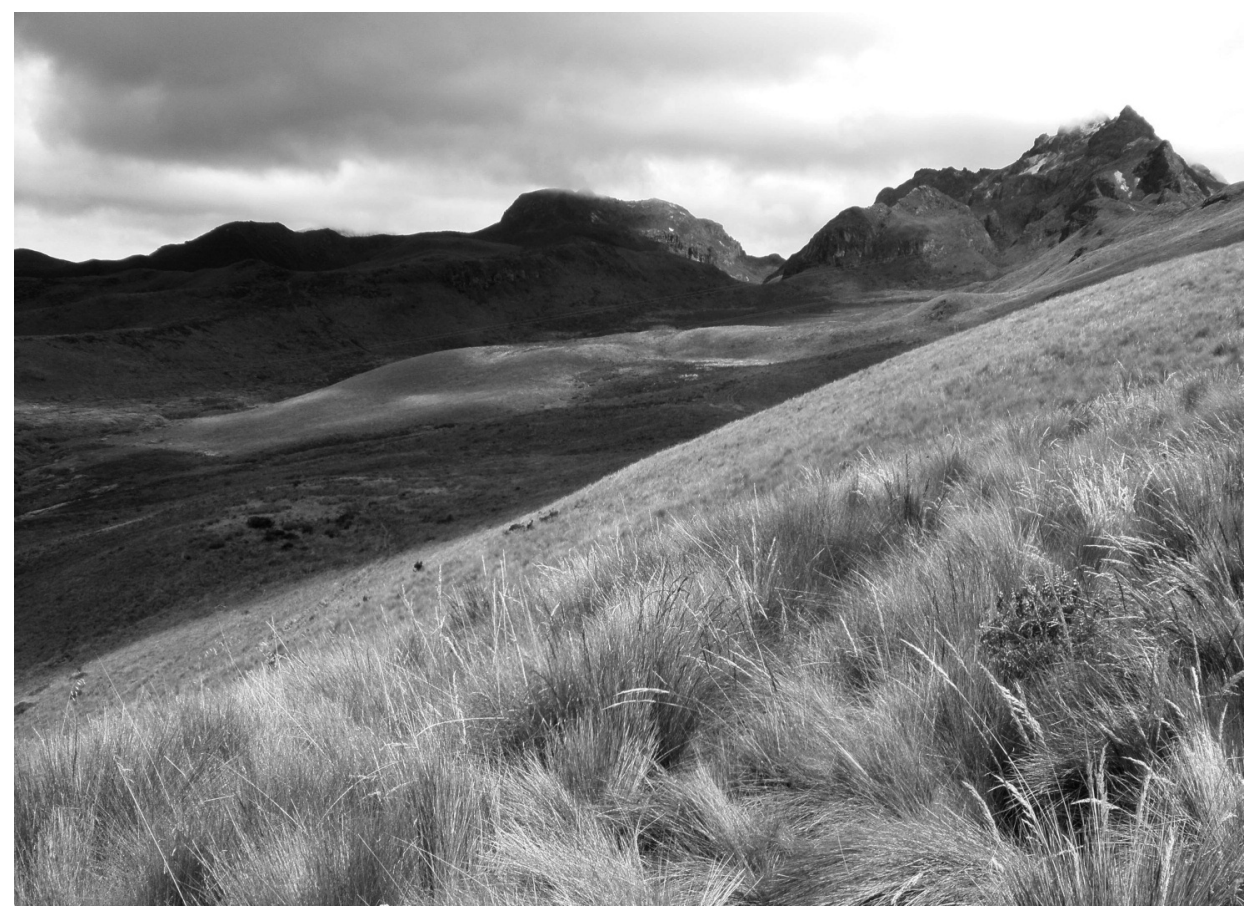

Nota: aspecto prototipo del páramo andino: predominio de formaciones herbáceas y relieves de grandes volúmenes (Rucu Pichincha, $\left.4.200 \mathrm{~m}, \lambda 0^{\circ} 10^{\prime} 35^{\prime \prime} ; \phi 78^{\circ} 33^{\prime} 06^{\prime \prime}\right)$.

Fuente: elaboración propia.

nezuela $(5,2 \%)$, en una corología disyunta que significativamente ha sido calificada como insular (Morales y Estévez, 2006). Los criterios más elementales de clasificación del páramo se refieren a la altitud y a la ausencia de formaciones forestales densas, aunque ambos aspectos no dejen de tener un carácter aproximado. Talmente la Ley de Conservación y Uso Sustentable de la Biodiversidad (título X), de Ecuador, fija la zona paramuna:

entre el actual o potencial límite superior del bosque andino cerrado y la línea de nieve perpetua, caracterizado por una vegetación dominante no arbórea, que incluye los fragmentos de bosque nativo propio de este ecosistema y que al norte del paralelo $3^{\circ} 00$ de latitud sur se encuentra sobre los 3.500 metros sobre el nivel del mar, y al sur de este paralelo sobre los 3.000 metros sobre el nivel del mar. 


\section{FIGURA 3}

\section{TIPOS DE VEGETACIÓN EN LOS ANDES EQUINOCCIALES}

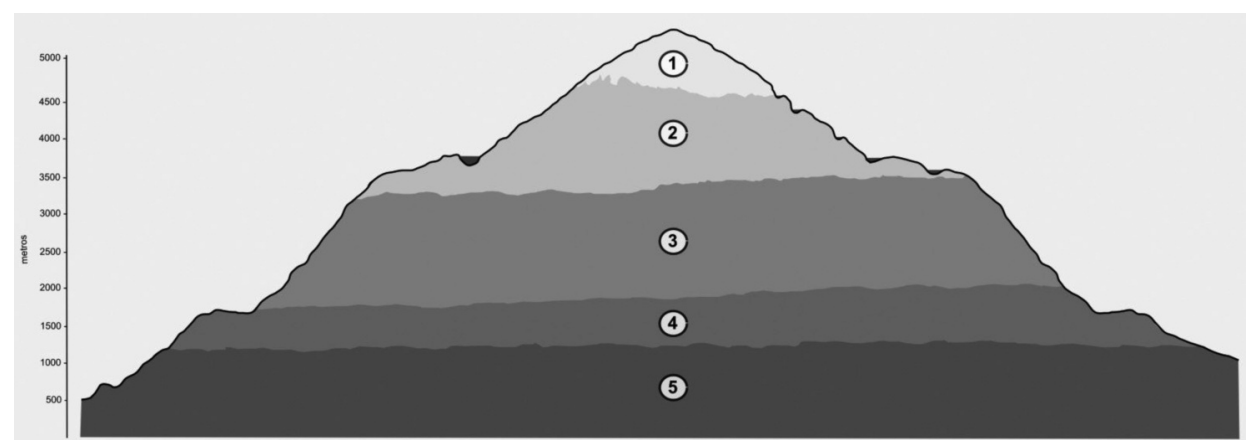

Nota: perfil idealizado de los grandes tipos de vegetación en las exposiciones orientales y occidentales de los Andes, a la altura del ecuador: 1: cubierta nivo-glacial; 2: páramo; 3: bosque altimontano; 4: bosque montano; 5: bosque y matorral basimontano.

Fuente: elaboración propia; datos modificados a partir de Sierra (1999) y Josse et al. (2009).

Pero estos valores resultan aproximados y a menudo se pueden detectar variaciones, como en Loja (aproximadamente a $2.800 \mathrm{~m}$, a $3^{\circ} 59^{\prime}$ ). En otras ocasiones la artificialización del páramo, o paramización, distorsiona completamente estos gradientes. El borde superior del páramo también resulta abierto. En determinadas ocasiones las masas glaciadas fijan una delimitación que, aunque fluctuante, resulta clara; pero en otras ocasiones éstas se hallan ausentes, y el páramo puede superar fácilmente los 4.500 metros (Josse et al., 2009). También la disimetría andina entre la cuenca amazónica y la pacífica introduce modificaciones importantes, pues la primera es mucho más húmeda que la segunda, donde se sienten los efectos secantes de la corriente fría de Humboldt (figura 3).

El páramo se encuentra indisociablemente ligado a los glaciares; incluso cuando éstos han desaparecido su antigua presencia continúa configurando su carácter (Morales y Estévez, 2006). La importancia de los glaciares andinos tropicales es máxima, pues su localización en latitudes bajas los hace extremadamente sensibles a las variaciones ambientales y, en consecuencia, los convierte en interesantes laboratorios a campo abierto (Francou y Vincent, 2007). De esta manera se estima que en Venezuela el área ocupada por glaciares disminuyó un 87\%, entre 1952 y 2003, mientras que en la Sierra Nevada colombiana la reducción de la masa glaciada fue cercana al $51 \%$, entre la década de 1950 y mitad de la del 2000. En Ecuador el glaciar del Cotopaxi se mantuvo 
estable entre 1956 y 1976, por bien que entre esta última fecha y 1997 perdió el 30\% de su masa, con una pérdida de volumen anual de 3-4 metros. En el Antisana la disminución del domo glaciar fue del 37\% y en el Chimborazo del 33\%, para el periodo 1979-2000. Finalmente, y a título indicativo, en el macizo de Huandoy-Artesonraju (Cordillera Blanca, Perú central) la pérdida fue de entre el 20 y el 30\%, entre 1962 y 2003. Con todo conviene puntualizar que las pérdidas, a pesar de ser constantes, no son lineales, tal como demuestra el mantenimiento o incluso crecimiento (por ejemplo, el Antisana 15 incrementó su lengua 43 metros en 1999-2000) de los glaciares durante los periodos húmedos marcados por La Niña (Rabatel et al., 2013).

En las líneas que siguen damos a conocer las características generales del páramo andino, un territorio de gran singularidad y enorme interés geográfico. Para ello adoptamos un planteamiento sistémico y fijamos interés en su dimensión ambiental, que es la que le concede una distinción más relevante. Mediante un ejercicio de síntesis repasamos las cuestiones de mayor significado en la configuración del páramo, prestando detenimiento a los servicios ambientales que ofrece y en la lectura geográfica que de ellos se deriva. Con esta contribución queremos dar a conocer a los geógrafos españoles un ámbito de trabajo de creciente interés entre nuestros colegas latinoamericanos.

\section{CONSTANTES TERRITORIALES}

\section{Contexto abiótico}

La característica climática que mejor define el páramo es la gran estabilidad anual de las temperaturas, debido a su localización en latitudes bajas, al tiempo que su elevada amplitud térmica diaria, explicada por la altitud a la que se encuentra (Troll, 1968). El contexto atmosférico general se describe a partir de la colisión de masas de aire húmedo y cálido de la costa y, particularmente de la Amazonía, con masas de aire frío de la Sierra.

De esta guisa resulta de gran interés la existencia de grandes contrastes térmicos de carácter diario y apenas ninguno de carácter anual. En la cota 3.300 se han cifrado medias mínimas de $3,9^{\circ}$ y máximas de $12,8^{\circ}$ (Keating, 2008) y en la cota 3.500 temperaturas medias alrededor de los $7^{\circ}$, si bien en las horas centrales del día se superan fácilmente los $30^{\circ}$ a pleno sol. La precipitación presenta valores más variables, tanto estacionales como espaciales. La temporada húmeda se alarga de septiembre a mayo, con episodios de lluvia típicos de alta frecuencia y baja intensidad. Con todo, el balance anual se demuestra bastante 
desigual. Por lo común se registran valores extremos entre 700 y $3.000 \mathrm{~mm}$; es por ello que algunos autores trabajan con rangos promedio de $1.200-2.000 \mathrm{~mm}$ (Luteyn, 1992; Buytaert et al., 2005). En otro orden conviene contemplar la precipitación horizontal, de notable frecuencia e intensidad, de la cual apenas se dispone de registros. La baja presión atmosférica, los vientos desecantes, la nitidez de la atmósfera y la perpendicularidad de los rayos del sol también comportan unos elevados valores de radiación e insolación, con una clara incidencia en el desarrollo de las formas de vida (Hedberg y Hedberg, 1979).

Experiencias realizadas en el páramo venezolano evidencian la dificultad de medir la evapotranspiración de forma fidedigna, a causa de factores ambientales como la humedad relativa, la radiación solar la oscilación térmica o la velocidad del viento (Maffei, 2012). Sin embargo se puede afirmar que el consumo de agua en el páramo es muy bajo porque, a pesar de que los valores de radiación sean elevados, las cubiertas vegetales autóctonas se componen de herbazales y pajonales de marcado carácter xerofítico. La evapotranspiración en esos ambientes se ha estimado entre 1 y $1,5 \mathrm{~mm} /$ día (Hofstede, 1995). Este hecho, unido a los altos volúmenes de precipitación, hace que el páramo sea claramente excedentario en agua (De Bièvre, Íñiguez y Buytaert, 2006). En efecto, las cabeceras de la red hídrica andina se nutren de manera prácticamente exclusiva de las aguas recogidas por el páramo. Estudios locales de balance hídrico indican que menos de un $1 \%$ del caudal de los ríos proviene de las aguas de fusión de glaciares, mientras que el resto corresponde en práctica exclusividad al páramo (De Bièvre et al., 2008). Aglomeraciones urbanas importantes, como Bogotá (más de siete millones de habitantes) o Quito (más de dos millones), se nutren de aguas reguladas por el páramo. Diversas actividades económicas dependen de forma directa de estas aguas, principalmente del sector primario y en particular la floricultura, que sólo en Ecuador se calcula que emplea entre 40.000 y 45.000 campesinos (Campaña, 2005). También la producción hidroeléctrica es notoria. Castrillón (2012) señala la importancia de las aguas del páramo para el abastecimiento de determinadas localidades colombianas, mientras que De Bièvre, Íñiguez y Buytaert (2006) reseñan el uso de las aguas en Ecuador, apuntando cuatro centrales hidroeléctricas dentro del páramo: Saucay (14.4 Mw), Saymirín (24 Mw), Pisayambo (70 Mw) y Paute (1100 Mw), a las que conviene sumar las que en 2014 aún estaban en construcción: Coca-Codo Sinclair (1500 Mw), Sopladora (487 Mw), ToachiPilatón (246 Mw) y Quijos-Baeza (100 Mw), entre otras.

El papel del páramo como regulador de agua se explica de manera muy especial por su estructura edáfica, que libera lentamente el agua almacenada durante los episodios de precipitación. Los suelos del páramo, excepto en el 
extremo meridional de su distribución, se caracterizan por la existencia de una capa homogénea de cenizas, proveniente de erupciones volcánicas cuaternarias (Sauer, 1957; Poulenard, Podwojewski y Herbillon, 2003), que según la clasificación de la FAO se designan como andosoles y, en aquellos lugares con mayor presencia de agua y menor de cenizas, histosoles. Estas formaciones tienen un elevado contenido de carbono orgánico, habitualmente de más del $10 \%$ y a menudo por encima del $40 \%$ (Podwojewski et al., 2002). Todo ello, unido a una estructura porosa y abierta y a una baja densidad aparente $(<0.9$ a 0.3) comporta que los suelos paramunos alberguen una retención de agua extraordinariamente alta (entre el $80 \%$ y el $90 \%$ ) y, que, atendiendo a la realidad topoclimática, esta capacidad potencial sea también real (Podwojewski y Poulenard, 2000).

\section{Contexto biótico}

Sin duda alguna el aspecto fisiognómico que mejor define al páramo es la vegetación natural o, incluso, la ausencia de ésta. Las formas vegetales propias del páramo son los herbazales de distinto tipo, comúnmente llamados pajonales. En determinados ambientes también aparecen formaciones arbustivas más o menos compactas, que acostumbran a desarrollarse de manera ufana llegando a superar los dos metros de altura (figura 4). De manera dispersa aparecen elementos arbóreos, pero siempre de forma aislada y muy raramente conformando bosquetes de cierta entidad (Sierra, 1999; Josse et al., 2009).

El páramo alberga elementos faunísticos de gran interés, como el oso de anteojos (Tremarctos ornatus), el zorro de páramo (Pseudalopex culpaeus) o el cóndor (Vultur gryphus), entre muchos otros. En conjunto se considera que son ecosistemas con valores de biodiversidad muy elevados y extraordinariamente singulares (Rangel, 2000; Castaño, 2002; Keating, 2008).

En este ambiente tan extremo las plantas desarrollan distintas estrategias a las condiciones de rusticidad, la más importante de las cuales seguramente sea el frío (Hedberg y Hedberg, 1979). La estrategia de adaptación que más comúnmente se ha invocado relaciona la talla de las plantas con su adaptación a las bajas temperaturas, de tal manera que las plantas de mayor talla crean a su alrededor un microclima que les permite evitar las heladas, mientras que las de talla más pequeña, que se hallan próximas al suelo, están más expuestas a temperaturas extremas y, por lo tanto, mejor adaptadas al frío. Sin embargo trabajos recientes señalan que esta correlación no es tan evidente como se suponía, y que la talla de las plantas no señala de forma inequívoca una mejor 


\section{FIGURA 4}

\section{ASPECTO DEL PÁRAMO HERBÁCEO}

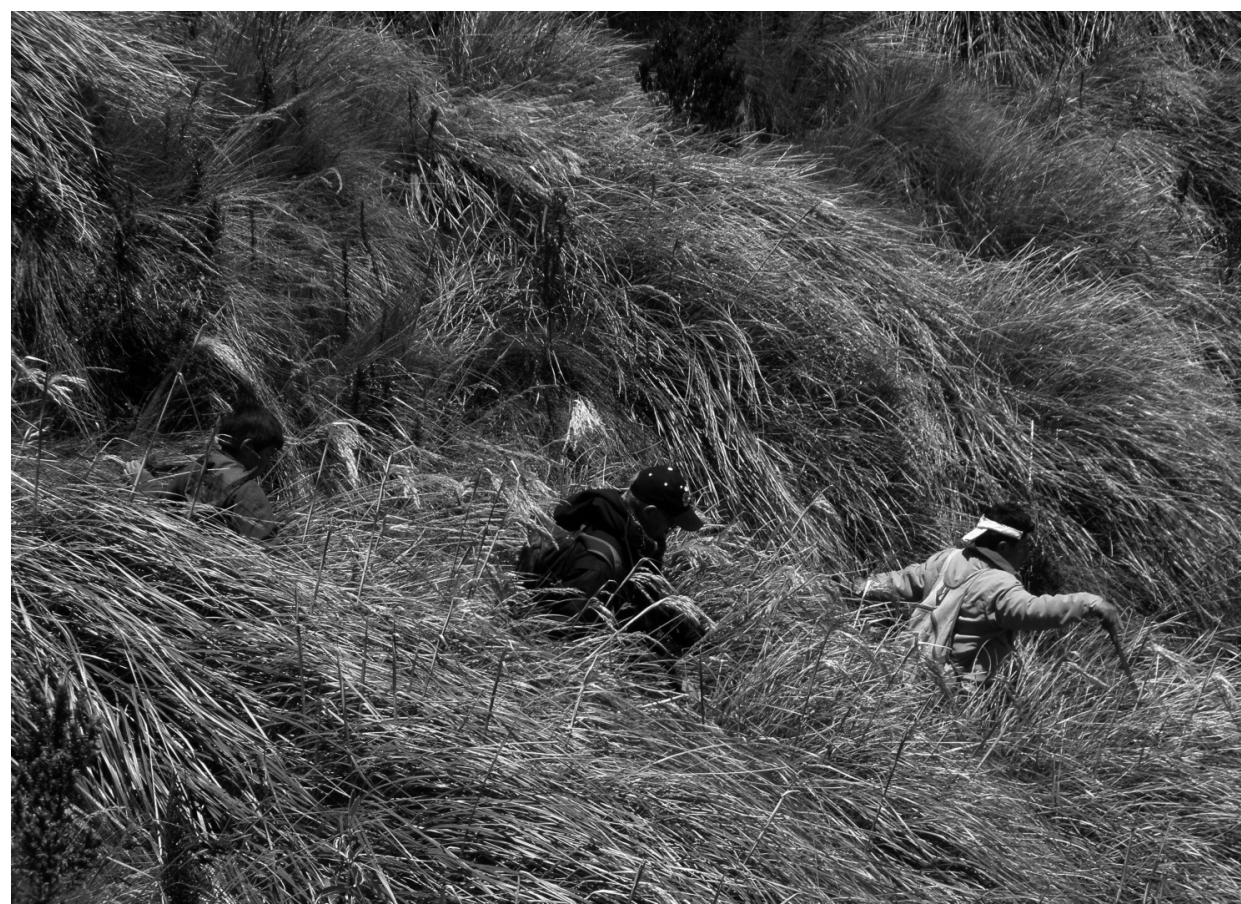

Nota: el páramo por antonomasia es el páramo herbáceo. En la imagen tres hombres entre formaciones de sigses (Cortaderia nitida), que superan en talla la de su cintura (nevado Cayambe, $4.000 \mathrm{~m}, \lambda 0^{\circ} 00^{\prime} 30^{\prime \prime} ; \phi$ $\left.78^{\circ} 01^{\prime} 57^{\prime \prime}\right)$.

Fuente: elaboración propia.

adaptación a las temperaturas deduciéndose, por tanto, la existencia de elementos de adaptación mucho más complejos (Sklenár et al., 2010). Estas estrategias, en general, han de ser comprendidas en clave de evolución en largas escalas temporales y tras una larga adaptación que debe entenderse a escala geológica (Graham, 2009).

Existen distintas clasificaciones de vegetación paramuna, según si se fija énfasis en la composición florística o la fisiognomía de las formaciones (Acosta Solís, 1968). Cuatrecasas (1958) divide el páramo en tres pisos: subpáramo, páramo y superpáramo, según la fisiognomía que adopta que, a la postre, deriva de la altitud en la que se encuentra. El WWF (2001) señala cua- 
tro tipos de páramos para el conjunto de los Andes, mientras que Sierra (1999) distingue doce formaciones, desglosables en cinco para la vertiente oriental y cinco para la occidental, más dos para las regiones más meridionales. Con todo, la extensión del páramo y las dificultades de acceso dificultan una tipificación del mismo (De Mesa, 2012). Las partes cimeras del páramo pertenecen a la llamada gelidofitia, que se emplaza a partir de los 4.700 metros y se caracteriza por la presencia de suelo desnudo salpicado de manchas de vegetación. En este espacio se encuentran musgos del género Andreana y Grimmia, y líquenes del género Lecanova y Gyrophora. La presencia de fanerógamas es escasa, y se representa por Aciachne flagellifera, Loricaria ferruginea, Draba aretioides y Valeriana pilosa (Acosta Solís, 1968). A partir de 4.200 metros, en la vertiente del Pacífico, se encuentra el páramo seco, caracterizado también por la alternancia de suelo desnudo y vegetación xerofítica. Aparecen gramíneas, arbustos y líquenes; destacan los táxones Azorella pedunculata, Chuquiraga jussieu, Hypochaeris sonchoides y Senecio microdon. En la vertiente amazónica, mucho más húmeda, esta formación se substituye por el páramo de almohadillas, que medra a partir de los 4.000-4.500 metros, según exposiciones (figura 5). Aquí la cobertura vegetal es mucho más densa, abundan distintos tipos de hierbas, arbustos, plantas en roseta basal y, en aquellos lugares más húmedos, las almohadilladas, como Azorella pedunculata, A. aretioides y A. corimbosa. También aparecen algunos árboles del género Polylepis, como $P$. pauta, $P$. incana o $P$. lanuginosa, que en los páramos colombianos ostentan un especial valor biogeográfico (Buitrago, Pulido y Vanegas, 2013).

En cotas inferiores, entre los 3.500-3.700 en la vertiente occidental y 3.000-3.500 en la oriental, aparece el páramo de frailejones, organizados por Espeletia pycnophylla, en densidades variables, pero que en ocasiones llega a conformar auténticos bosques. Los frailejones alcanzan una altura superior a los 3 metros y, en la vertiente oriental, hasta de cinco. Su distribución es pródiga en Venezuela y Colombia (Castaño, 2002), y más rara en Ecuador, donde aparece en el norte, desaparece por el centro del país y vuelve a encontrarse de forma tímida en Llagantes (Tungurahua); otras especies que medran en este piso son Oreopanax sodiroi, Eriocaulion microcephallum y el género Azorella. A partir de los 3.400-4.000 metros se encuentra el páramo herbáceo, que es el que habitualmente se considera páramo por excelencia y el que ocupa mayor superficie. Se caracteriza por la alta densidad de la cubierta vegetal, prácticamente dominada por hierbas del género Festuca y Calamagrostis, así como Hypochaeris y Baccharis. Destacan las morfologías con aspecto de penacho que, dada la considerable altura de los tallos, tiende a doblegarse y aumentar el aspecto de alta densidad de la formación. Su límite inferior co- 


\section{FIGURA 5}

FORMACIONES ALMOHADILLADAS

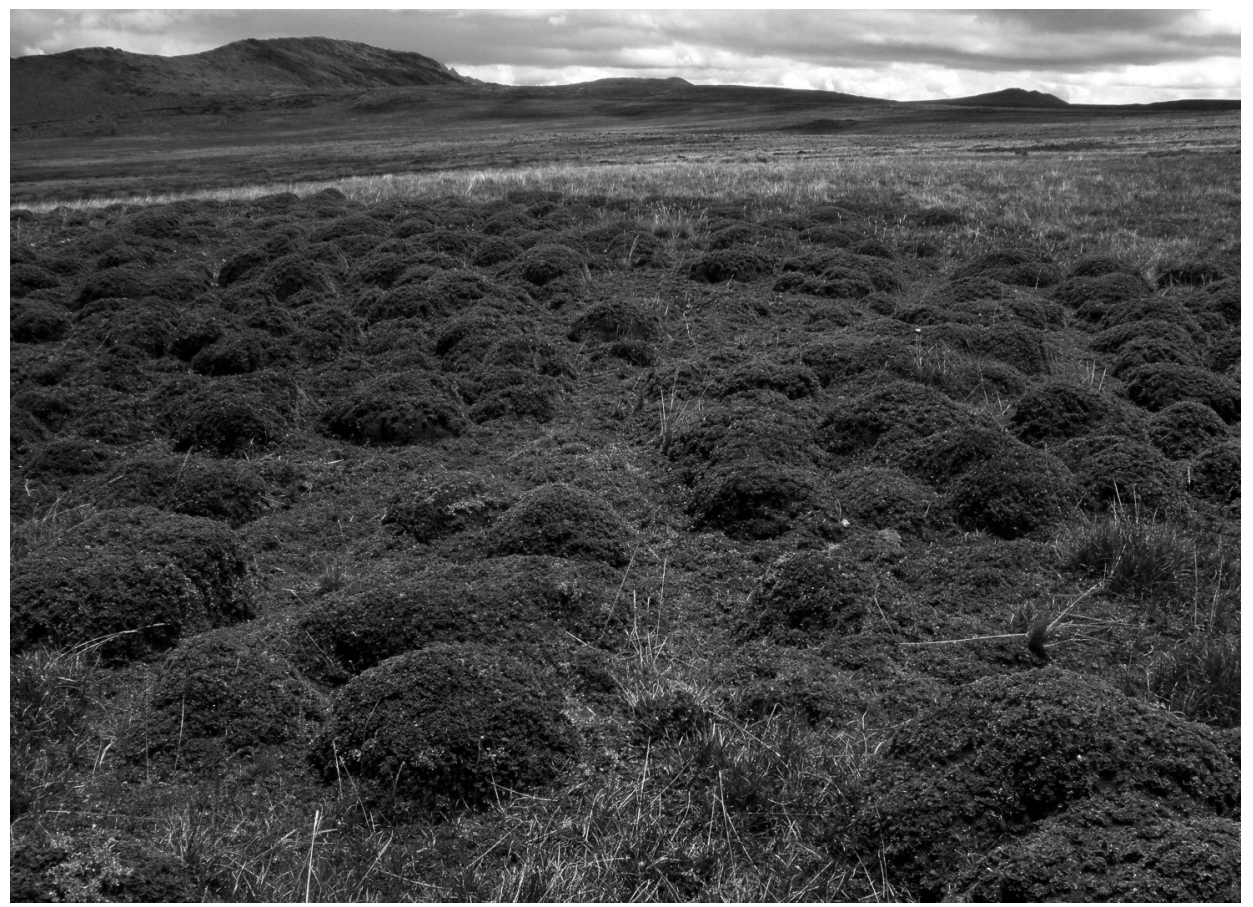

Nota: formaciones almohadilladas (primer plano) y herbáceas (segundo plano) en la laguna de la Mica (Antisana, 3.900m, $\left.\lambda 0^{\circ} 32^{\prime} 21^{\prime \prime} ; \phi 78^{\circ} 12^{\prime} 07^{\prime \prime}\right)$.

Fuente: elaboración propia.

incide con la denominada por Acosta Solís (1966) ceja andina, y se refiere al ecotono formado por el límite superior del bosque andino y el subpáramo. Aparte es aún posible distinguir un herbazal lacustre montano, en emplazamientos variables, por lo común por encima de los 2.100 metros y alrededor de lagunas y otros cuerpos de aguas tranquilas, donde domina la planta endémica Elatine ecuadoriensis (Sierra, 1999). No obstante la corología típica de los pisos de vegetación, hay que tener en cuenta la complejidad topográfica y microclimática del páramo, que introduce importantes modificaciones a nivel local y, en consecuencia, cambios significativos en la distribución zonal de la vegetación, tal como ya se ha demostrado en el páramo venezolano (Nidia y Cleef, 2009). 
SERVICIOS AMBIENTALES DEL PÁRAMO

\section{Sobre los aprovechamientos hídricos}

La capacidad del páramo para almacenar y regular agua ha sido estudiada en distintas ocasiones (Hofstede, Chilito y Sandoval, 1995; Harden, 2006). A causa de las dificultades existentes para extraer agua del subsuelo, las aguas de escorrentía son las más aprovechadas del páramo. Es por este motivo que cualquier cambio en la ocupación del suelo puede afectar sensiblemente su balance hídrico. Un aspecto importante en la regulación hídrica del páramo son las quemas de pasto. Con el fin de conseguir mejores pastos para el ganado, numerosas superficies de páramo son periódicamente incendiadas, favoreciendo la aparición de brotes tiernos de fácil acceso a los rumiantes (Hofstede, 1995). Los efectos de estos incendios en la biodiversisad son obviamente notables, pero no así en las propiedades del suelo. A diferencia de los suelos existentes en otros ambientes, como por ejemplo los de carácter mediterráneo, los suelos paramunos se encuentran permanentemente húmedos, y rara vez sienten las consecuencias de la quema más allá de los centímetros más superficiales. Con todo, cabe mencionar que la recuperación edáfica es muy lenta (Camargo-García et al., 2012). Únicamente en aquellos casos en los que las quemas adquieren carácter recurrente, o bien están precedidas de actividades agrícolas, se observan procesos erosivos y pérdidas en la capacidad para retener agua (De Bièvre, Íñiguez y Buytaert, 2006).

Por otra parte conviene hacer especial referencia a las plantaciones forestales. Particularmente aquellas realizadas con especies exóticas, como el pino o el eucalipto, producen una merma en la disponibilidad de agua, con descensos de hasta el 70\% (Buytaert et al., 2008). Las superficies con formaciones arboladas naturales, sin embargo, ofrecen valores distintos, especialmente porque los árboles parecen en distinto grado de madurez, se encuentran formados por distintas especies y, particularmente, porque presentan una adaptación metabólica a una mayor eficiencia hídrica.

\section{Sobre el origen herbáceo del páramo}

El carácter herbáceo del páramo, su origen natural y el límite superior del bosque ha sido discutido en numerosas ocasiones, debatiéndose hasta qué punto el aspecto actual del páramo ha sido estable a lo largo del tiempo (Van 
der Hammen, 1997; Luteyn, 1992; Varela, 2008). Distintos análisis polínicos realizados en el norte de Perú y el sur de Ecuador han ayudado a clarificar la evolución del páramo a lo largo de los últimos milenios y, en la medida de lo posible, el inicio de su antropización.

Se considera que para el último máximo glacial, hace aproximadamente unos 20.000 años, las condiciones ambientales eran mucho más frías y húmedas que las actuales. En este periodo abundan restos polínicos de la familia Poaceae y de Plantago rigida, el páramo ocupaba una extensión superior a la actual y el límite superior del bosque se encontraba en cotas inferiores a las de hoy en día. Se ha calculado que el borde forestal se fijaba unos $700 \mathrm{~m}$ más abajo que el actual en exposiciones norte, y unos 250 metros en exposiciones sur, considerando siempre la complejidad topográfica de la cordillera andina (Brunschön y Behling, 2010). En unos 16.200 años hubo un incremento de las temperaturas, hecho que se evidencia en un ascenso de la superficie forestada y en una elevación del límite superior del bosque. Este crecimiento se dio particularmente con abundantes elementos de la familia Melastomataceae, Hedyosum y, en menor medida Polypepis, tal como evidencian los restos polínicos. Muy posiblemente en esta época se marca el máximo ascensional del bosque, que cabría situar unos 200 metros por encima del actual. Hace 8.500-4.300 años el dominio del páramo se incrementa, las masas forestales disminuyen y se produce la extinción de algunos géneros de Polypepis; en estas condiciones más frías y secas aumenta el dominio de las poáceas. En torno 1.800 años atrás la superficie ocupada por páramo decrece ligeramente, situándose probablemente en una cota similar a la de hoy en día. En todos los registros estudiados apenas se delatan elementos de antropización. Los primeros valores significativos de cenizas datan de alrededor 8.000 años, pero no es hasta hace unos 2.300-1.000 años que toman mayor recurrencia. Es precisamente este dato el que ha llevado a sugerir que la antropización del páramo es relativamente reciente, al menos en comparación con otros ambientes andinos (Brunschön y Behling, 2009; Rodríguez y Behling, 2012; Villota, León-Yánez y Behling, 2012).

\section{Sobre la necesidad de forestar}

Desde la década de 1920 las tierras del páramo han sido objeto de numerosos ensayos forestales, que tenían por objeto incrementar la masa arbolada de estos parajes (Farley, 2008). Este esfuerzo forestal aumentó de manera sensible a partir de la década de 1960, materializándose siempre con especies exóticas, como el pino de Monterrey (Pinus radiata), el pino ocote (Pinus patula) o 
el eucalipto (Eucalyptus globulus). El porqué de las plantaciones a menudo se ha justificado por la obtención de madera, la producción de bienes y servicios, el empleo de mano de obra, la utilización de tierras de baja productividad y, en los últimos años, el almacenamiento de carbono. Con todo bajo estas distintas argumentaciones subyace la idea que el páramo, desarbolado, carece de valor y que, por el contrario, cualquier superficie forestada lo incrementa, sin llegar a precisar de qué tipo de valor se trata (Farley, 2007).

Los efectos biofísicos esperables de las reforestaciones acostumbran a centrarse en el incremento de la calidad y cantidad de agua, la mejora de la calidad del suelo, la protección contra la erosión y el almacenamiento de carbono. Sin embargo, las características peculiares del páramo hacen que estos efectos no siempre se cumplan de la forma en que acostumbran en otros ambientes. Así, los estudios centrados en regulación de agua no siguen las pautas habituales. En el Cotopaxi, por ejemplo, se estimó que los suelos forestados con pinos retenían entre un 39\% y un 63\% menos de agua que los del pajonal, como consecuencia del incremento de la evapotranspiración, a través de las hojas, y un mayor acceso al agua del subsuelo, a través de las raíces (Farley y Kelly, 2004); en otras cuencas experimentales de Ecuador los valores de evapotranspiración también son muy elevados y los balances hídricos anuales resultan claramente inferiores a los de superficies ocupadas por pajonal (Buytaert et al., 2008; Crespo et al., 2010). En cuanto a procesos edáficos se ha comprobado que en suelos degradados las plantaciones forestales mejoran la cantidad de carbono retenido, si bien en suelos ricos en carbono, como los del páramo, el efecto es inverso. En los Andes centrales se ha observado una pérdida de $5 \mathrm{~kg} / \mathrm{m}^{2}$ de carbono en el suelo, que pasó a localizarse en el arbolado produciéndose, no un incremento, sino una redistribución del mismo (Farley y Kelly, 2004). De forma similar, y en cuanto a fertilidad y propiedades químicas, en los primeros 10 centímetros de plantaciones de pinos, se ha detectado un $\mathrm{pH}$ sensiblemente más ácido que en páramos cubiertos por herbazales. Este hecho hace sospechar la influencia de los ácidos orgánicos de la pinaza durante el proceso de humificación, y ha sugerido a algunos autores (Chacón, Gagnon y Paré, 2009) la oportunidad de establecer plantaciones sobre suelos degradados, pero no sobre suelos bien conformados, por los efectos adversos que se derivan. En cuanto a restauración y protección de cuencas hidrográficas, se han obtenido resultados bastante desiguales. Por lo general se admite que las superficies forestadas mitigan los efectos atmosféricos y ejercen una acción amortiguadora y protectora sobre los suelos donde se asientan. Los estudios desarrollados en páramos colombianos sobre vegetación arbolada autóctona así lo demuestran, señalando una preservación de la humedad y una 
protección evidente frente a vientos desecantes (Buitrago, Pulido y Vanegas, 2013). Por el contrario en determinadas ocasiones las plantaciones tuvieron efectos adversos, especialmente si se realizaron con especies alóctonas (Podwojewski y Poulenard, 2001; Medina y Mena, 2000; Chacón, Gagnon y Paré, 2009). En otros casos, finalmente, no se detectaron problemas ambientales significativos, al contrario, las plantaciones ayudaron a mejorar la estructura del suelo, mitigar procesos erosivos y fomentar el establecimiento de vegetación autóctona, incluso de tipo arbóreo (Hofstede et al., 2002).

\section{Sobre la fijación de carbono}

El páramo también ha sido entendido como un gran sumidero de carbono, no tanto por la biomasa que sustenta, como por las formaciones edáficas que alberga. En efecto, el pajonal del páramo puede cuantificarse en unas 40tn/ha, como máximo, de materia seca, lo que equivale a unas 20tn/ha de carbono. Sin embargo en el páramo los procesos de humificación son muy lentos, a causa de las bajas temperaturas, la alta humedad y la existencia de cenizas volcánicas. Es por ello que los suelos paramunos alcanzan potencias de gran magnitud (hasta dos metros, con facilidad), donde se constatan concentraciones de carbono altísimas, de hasta 1.700tn/ha si la densidad aparente del suelo es la propicia (Hofstede, 1999). Es precisamente este aspecto el que diferencia la capacidad de almacenar carbono del páramo de la capacidad de la selva amazónica, ya que aunque en ésta la biomasa es mucho mayor (unas 500tn/ha de materia seca), las condiciones de elevada temperatura y humedad comportan una rápida descomposición de la materia orgánica, que en muchos casos se incorpora al suelo oxidada y, por lo tanto, no permite la creación de un verdadero horizonte orgánico.

La ubicación del páramo, más cerca de grandes núcleos urbanos que de la selva, su baja productividad agrícola y la ausencia de cubierta arbolada, a menudo han motivado su forestación y, en los últimos años, utilizando como pretexto la fijación de carbono (Farley, 2008). Pero la capacidad de fijar carbono por parte de plantaciones forestales es muy desigual. Según en qué localidades se trabaje, qué especies se utilicen y qué técnicas de plantación y mantenimiento se apliquen se obtienen valores en un sentido u otro. Es por ello que, en este sentido, se aconseja actuar con máxima prudencia pues, de hecho, la simple remoción del suelo del páramo comporta su desecación y, por tanto, un aumento de la descomposición, la oxidación y, en consecuencia, las emisiones de carbono a la atmósfera (Hofstede y Aguirre, 1999; Henry et al., 2012). 


\section{Sobre el aprovechamiento social}

Aparte de todos los aspectos ambientales que puedan reseñarse, el páramo es, sin duda, un espacio social (Ramón, 2002; Girard, 2005). Un espacio social que genera beneficios económicos, que ayuda a configurar entidades y que conforma un carácter determinado al territorio. En las últimas décadas se han producido distintos cambios, que están dibujando el páramo como un espacio de marcada identidad (figura 6).

Los cambios con un significado fisiognómico más destacado se relacionan siempre con las actividades primarias. Desde las cotas bajas, donde se concen-

FIGURA 6

\section{DISTRIBUCIÓN DEL PÁRAMO}

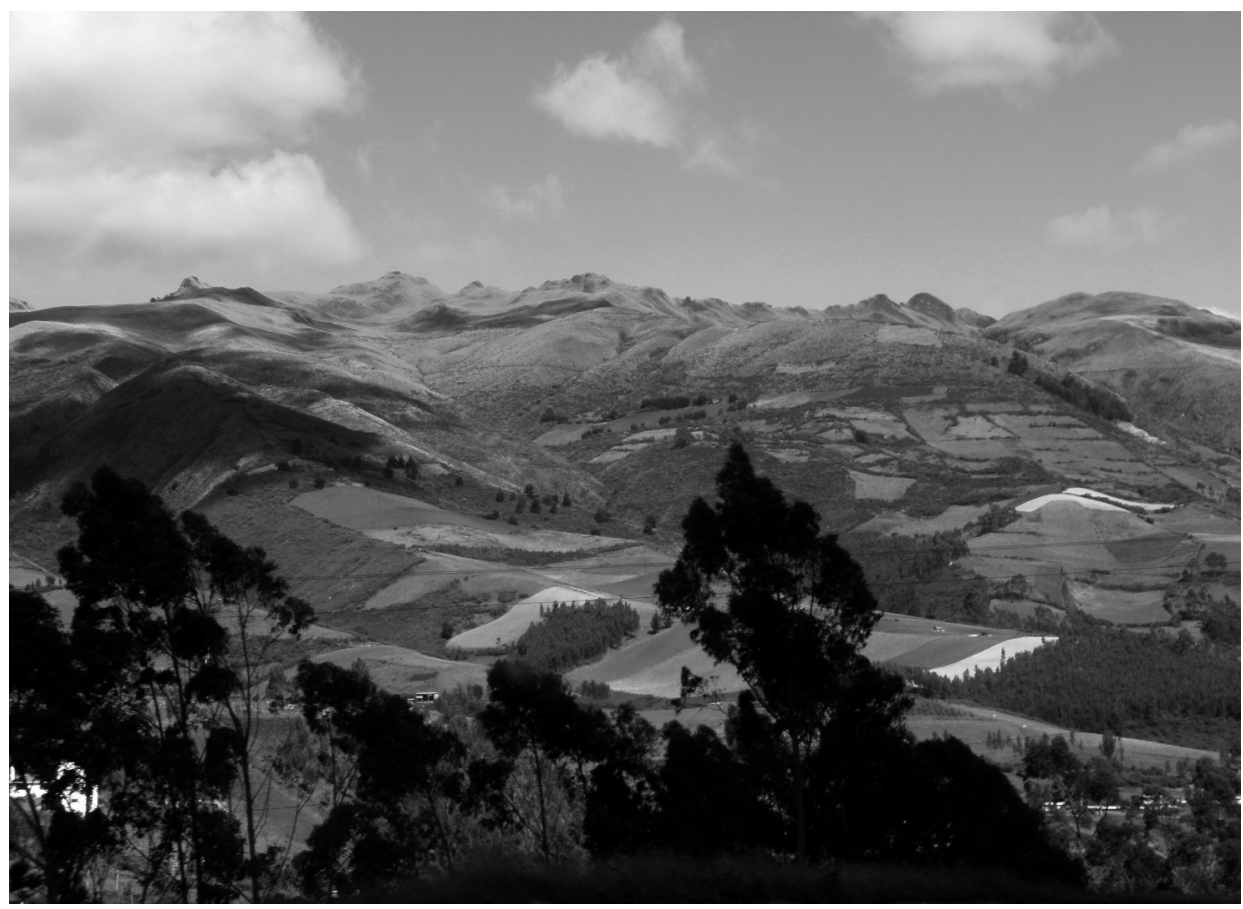

Nota: la distribución natural del páramo cubriría la mayor parte de las elevaciones montañosas. En la actualidad las cotas bajas aparecen ocupadas por áreas de cultivos y pastos, y únicamente en los bordes de mayor altitud aparecen cubiertas originarias (Imbabura, $\lambda 0^{\circ} 15^{\prime} 03^{\prime \prime} ; \phi 78^{\circ} 13^{\prime} 30^{\prime \prime}$ ).

Fuente: elaboración propia. 
tran la mayor parte de tierras de cultivo y aprovechamientos económicos (Campaña, 2005), se observa una alteración del páramo y un incremento en altitud de las tierras de labor y pastoreo. De esta manera se producen cambios en la ocupación del suelo, particularmente por la aparición de potreros, o tierras de pasto para ganado vacuno, y también por la apertura de tierras para agricultura, donde medran bien diversas raíces y tubérculos adaptados a condiciones extremas, como el melloco, la oca, el cúbio o mashua, el olluco, el yacón o la zanahoria blanca, aparte de una gran variedad de papas y camotes. En Colombia, por otra parte, hay que considerar también los cultivos ilícitos, de muy difícil cuantificación y localización.

El páramo también es objeto de aprovechamiento ganadero, particularmente vacuno. Con otro carácter, y fijando más atención en la degradación ambiental que en la sobrecarga ganadera, hay que referirse también a la presencia de rumiantes salvajes y domésticos de cría extensiva. De los cuatro camélidos andinos que existen, tres son pobladores del páramo: el guanaco (Lama guanicoe) se distribuye ampliamente por el cono sur y la puna, sin llegar a superar en su área de distribución natural el centro de Perú y, por lo tanto, sin atender en sentido estricto al páramo. Sin embargo, la llama (Lama glama), pariente domesticada del guanaco fue utilizada desde épocas incaicas como animal de carga y para la obtención de fibras textiles, hecho que amplió su área natural de poblamiento hacía el norte, alcanzando territorios paramunos. La vicuña (Vicugna vicungna) habita en estado silvestre en el páramo, mientras que la alpaca (Vicugna pacos) es una especie domesticada a partir de la vicuña y la llama, con abundante presencia en el páramo por el interés comercial de su lana. De todos estos ungulados solo la vicuña se reconoce que tiene un bajo impacto en el medio, porque sus pezuñas son almohadilladas y sus pisadas mitigan los procesos de erosión, hecho que no pasa con los otros camélidos (Medina y Mena, 2001). Bajo esta coyuntura se están articulando distintas iniciativas que buscan optimizar estos recursos de la manera más sostenible posible. Así destaca en Ecuador la iniciativa Alpa-Huasy, que nace de una empresa peruana (Ecovalley) y una ecuatoriana (Ecoffice), y tiene por objetivo promover la cría de alpacas con fines industriales, pero de manera armonizada con el medio. Trabaja en la zona de amortiguamiento del parque nacional del Cotopaxi y tiene por objetivo criar y procesar la lana de alpaca para la confección de prendas de vestir y accesorios de forma no agresiva con el torno, revertiendo parte de sus ganancias en la población local (Solórzano, 2005).

Una realidad cada vez más frecuente en toda la región, pero particularmente en Ecuador, es el crecimiento y consolidación del mercado turístico que, prácticamente de forma natural, se está convirtiendo en ecoturístico. Un 
caso interesante es el de Oyacachi, una comunidad de indígenas quichuas en la reserva ecológica Cayambe-Coca. Se trata de una comunidad pequeña, de unas 400 personas organizadas en 120 familias dedicadas a la agricultura, la ganadería, la elaboración de artesanías y, recientemente, al turismo. El principal reclamo del lugar es la belleza escénica y las aguas termales, que se encuentran explotadas mediante un proyecto comunitario. Aunque el censo de visitantes no es exhaustivo, se conocen datos fiables para 1999, que cifraban en aproximadamente 10.000 el número de turistas, y para 2004, que lo cuantificaba en alrededor de 20.000. Se trata de una tendencia claramente al alza, que en torno a un $80 \%$ se cifra en turismo doméstico, a menudo proveniente de otras destinaciones cercanas, como Cangahua, Cayambe u Otavalo. Desde la misma comunidad surgió la necesidad de organizar y optimizar los recursos disponibles, de tal manera que, de forma consciente y no forzada, se ha ido fortaleciendo el sector turístico. Esta promoción eminentemente endógena se ha construido con la adecuación para su visita de los restos arqueológicos de Maucallacta, la promoción de la gastronomía y las artesanías, añadiendo valor y diversificando las visitas a las aguas termales. De forma prácticamente natural se ha generado y favorecido un perfil turístico de mayor nivel social y adquisitivo y, sin existir paquetes turísticos, se ha actuado como si los hubiera favoreciendo las estancias de dos días con una pernoctación. También se han buscado atractivos en el medio natural, hecho que ha provocado una selección, prácticamente inconsciente, del perfil del visitante, que cada vez presenta mayor sensibilidad ambiental. De un tiempo a esta parte los oyacacheños jóvenes ven en el turismo una alternativa de vida real en su comunidad, hecho que ha favorecido su organización y la aparición de estudios de viabilidad, mercado, factibilidad financiera y capacidad de carga, siempre con miras al turismo. Se han realizado planes de acción, se ha mejorado la infraestructura hotelera y de restaurantes, se ha diseñado un logo y se han editado folletos turísticos, así como un portal web. Pero lo más significativo de este proceso es que no solo se ha generado de forma espontánea, sino que, además, ha nacido y se ha conformado en el seno de la comunidad, con la participación voluntaria de sus miembros y sin dependencias de carácter externo (Flores y Parión, 2005).

\section{Sobre políticas públicas}

Posiblemente la mejor iniciativa internacional para la protección del páramo sea el Proyecto de conservación de la biodiversidad del páramo en los 
Andes del norte y centrales (PPA). Se trata de una iniciativa que reúne a Venezuela, Colombia, Ecuador y Perú, se encuentra enmarcado por el Global Environmental Found y administrado por el PNUMA. La coordinación entre los cuatro países va a cargo del Consorcio para el desarrollo sostenible de la ecorregión andina (CONDESAN), al que se suman el Instituto de Ciencias ambientales y ecológicas de los Andes, en Venezuela; el Instituto Alexander von Humboldt, en Colombia; EcoCiencia, en Ecuador; y el Instituto de Montaña, en Perú (Mena, 2004). Aparte resulta oportuno señalar distintas contribuciones de carácter internacional, particularmente desde Europa. En este sentido destacan de manera especial las promulgadas por la sede del UICN en los Países Bajos, y las canalizadas directamente por la administración de este país (Ferweda, 2001). Otras iniciativas, como el Grupo de trabajo del páramo, ayudan a establecer canales de comunicación y mecanismos de trabajo conjunto a nivel internacional (Hofstede y Mújica, 2002). La cooperación internacional, tanto en el ámbito gubernamental como no gubernamental, toma especial importancia en un ecosistema de tales características y dimensiones. Una buena manera de evidenciar este proceso es a través de los espacios naturales protegidos. Aunque la verdadera función de éstos siempre queda supeditada a la gestión que se realice, tal como se ha demostrado en Colombia (Morales y Estévez, 2006).

De un tiempo a esta parte se han creado distintas instituciones que tienen por objeto velar por el buen uso del agua del páramo. Posiblemente la más renombrada de todas sea el Fondo para la Protección del Agua (FONAG), fundado en 2000 en la ciudad de Quito. El FONAG se ha convertido en una institución pionera en la gestión del agua mediante mecanismos de voluntariado, con procedimientos descentralizados y apoyo a los agentes locales. Desde su aparición 15 instituciones similares más han nacido en el área andina, siete de las cuales radican en Ecuador, con dinámicas y modelos de gestión de carácter parecido (Kauffman y Echavarría, 2012).

Con todo, el páramo es un espacio con un tejido cultural muy denso. Es por ello que, sea cual sea la acción que quiera desarrollarse, tal como ya se ha evidenciado (Ramón, 2002), para que llegue a buen término, resulta de vital importancia el compromiso de la población local. La participación e implicación de las comunidades indígenas asegura que los proyectos a desarrollar, no solo se encaucen correctamente, sino que, además, cumplan una función pedagógica (Barros y Proaño, 2011). 


\section{CONSIDERACIONES FINALES}

El páramo andino es un espacio de la alta montaña tropical con un elevado valor cultural y ambiental. Se distribuye mayormente en Ecuador y Colombia, con pequeñas contribuciones en Perú y Venezuela. Su emplazamiento a una gran altitud en las latitudes bajas del planeta le confiere una fisiognomía y un funcionamiento bien particular. Las condiciones climáticas extremas del páramo han originado una adaptación de las formas de vida que lo pueblan, que se materializa de forma evidente a través de la cubierta vegetal, predominantemente herbácea y, en menor medida, arbustiva. Y se materializa también en una fragilidad ecológica notoria, que se revela de máxima importancia cuando la sociedad intenta beneficiarse de los recursos que ofrece.

De esta manera el páramo almacena y regula los recursos hídricos de las cotas altas de los Andes, y nutre de agua la red fluvial de los territorios aledaños. Determinados cambios en la ocupación del suelo afectan de forma evidente esta capacidad reguladora. De tal forma la instalación de nuevas tierras de cultivo, el establecimiento de plantaciones forestales y, en general la remoción de la capa edáfica paramuna comporta una disminución del balance hídrico y, a la postre, una merma de las aguas de escorrentía. En este contexto resulta de especial interés el papel de las plantaciones forestales, que particularmente en el caso de especies exóticas, actúa de manera contraria a como acostumbra a operar en otros ámbitos. También resulta interesante el papel de los suelos del páramo que, a causa de las condiciones ambientales en que se origina, alberga un contenido en carbono extraordinariamente alto, pero también fácilmente degradable si, por ejemplo, se abren nuevos campos de cultivo. El páramo, en efecto, acoge numerosos aprovechamientos sociales y económicos, que se expresan en forma de distintas actividades primarias y, más recientemente, a través de la actividad turística. De un tiempo a esta parte los valores y servicios ambientales del páramo han sido reconocidos, tanto por la administración pública como por distintos movimientos ciudadanos y académicos. Una muestra de ello es el establecimiento de espacios naturales protegidos, así como la conformación de redes de conocimiento y trabajo, y también las políticas específicas que cuentan con la población implicada.

A partir de esta coyuntura, y atendiendo a la consideración que el páramo despierta entre los geógrafos latinoamericanos, esta aportación pretende colaborar a la creación un marco de trabajo general que sirva de punto de partida para futuros trabajos de los geógrafos, ahora sí, iberoamericanos.

Fecha de recepción: 11 de febrero de 2014.

Fecha de aceptación: 8 de octubre de 2014. 
BiBLIOGRAFÍA

Acosta Solís, M. (1966): "Las divisiones fitogeográficas y las formaciones geobotánicas del Ecuador". Revista de la Academia Colombiana, 12, pp. 401-447.

Acosta Solís, M. (1968): Divisiones fitogeográficas y formaciones geobotánicas del Ecuador. Quito, Casa de la Cultura Ecuatoriana.

Ange, C. (ed.) (2002): Congreso mundial de páramos. vol. II. Bogotá, Ministerio de Medio Ambiente, 204 pp.

Barros, R. y Proaño, M. (2011): "Una plataforma social para la gestión de los páramos en la subcuenca del río San Pedro en el cantón Mejía, Pichincha, Ecuador". Urku Yaku Wachariy, 1, pp. 43-55.

Brunschön, C. y Behling, H. (2009): "Late quaternary vegetation, fire and climate history reconstructed from two cores at Cerro Toledo, Podocarpus National Park, southeastern Ecuadorian Andes". Quaternary Research, 72, pp. 388-399.

Brunschön, C. y Behling, H. (2010): "Reconstruction and visualization of upper forest line and vegetation changes in the Andean depression region of southeastern Ecuador since the last glacial maximum - A multi-site syntesis". Review of Palaeobotany and Palynology, 163, pp. 139-152.

Buitrago, S. P.; Pulido, K. L. y Vanegas, L. J. (2013): “Environmental variability and physiological responses from Polylepsis cuadrijuga (Rosaceae) in fragmented environment in the páramo de la Rusia (Colombia)". Revista de biología tropical, 61/1, pp. 351-361.

Buytaert, W.; De Bièvre, B.; Célleri, R.; Cisneros, F; Wyseure, G. y Deckers, S. (2008): "Comment on «Human impacts on headwater fluvial systems in the northern and central Andes» (Carol P. Harden, Geomorphology 79, 249-263)”. Geomorphology, 96, pp. 239-242.

Buytaert, W.; De Bièvre, B.; Wyseure, G. y Deckers, J. (2005): "The use of the linear reservoir concept to quantify the impact of land use change on the hydrology of catchments in the Ecuadorian Andes". Hydrology and Earth System Sciences, 8, pp. 108-114.

Camargo-García, J. C.; Dossman, M. Á.; Rodríguez, J. A.; Arias, L. M. y Galvis-Quintero, J. H. (2012): "Soil changes after a fire event in a paramo ecosystem: Los Nevados natural national Park, Colombia". Acta Agronomica, 61/2, pp. 151-165.

Campaña, A. (2005): "Floricultura: Algunos aspectos de su impacto humano y ambiental”. Páramo, 20, pp. 25-30.

Castaño, C. (2002): Páramos y ecosistemas alto andinos de Colombia en condición hotspot y global climatic tensor. Bogotá, Editorial IDEAM, 387 pp.

Castrillón, E. (2012): "The Páramo ecosystems, wetlands, and oak forests in the river basin of Rio Grande in Antioquia (Colombia) in a proposal for sustainable water management", en ASABE (ed.): American Society of Agricultural and Biological Engineers Annual International Meeting, 2012. Saint Joseph, pp. 4282-4294. 
Chacón, G.; Gagnon, D. y Paré, D. (2009): “Comparision of soil properties of native forests, Pinus patula plantations and adjacent pastures in the Andean highlands of southern Ecuador: Land use history or recent vegetation effects?" Soil use and Management, 25, 4, pp. 427-433.

Crespo, P.; Célleri, R.; Buytaert, W.; Feyen, J.; Íñiguez, V.; Borja, P. y De Bièvre, B. (2010): "Land use change impacts on the hydrology of wet Andean páramo ecosystems", en IAHS-AISH Publication: International Workshop on status and perspectives of hydrology in Small Basins, pp. 71-76.

Cuatrecasas, J. (1958): "Aspectos de la vegetación natural de Colombia". Revista de la Academia colombiana de ciencias exactas, físicas y naturales, 10, pp. 221-264.

De Bièvre, B.; Coello, X.; De Keizer, O. y Maljaars, P. (2008): Modelo hidrológico de la Hoya de Quito. Proyecto Manejo Integrado de los Recursos Hídricos en la Hoya de Quito. Quito, UICN-Sur, 64. pp.

De Bièvre, B.; Íñiguez, V. y Buytaert, W. (2006): "Hidrología del páramo. Importancia, propiedades y vulnerabilidad". Páramo, 21, pp. 26-44.

De Mesa, J. (2012): "Remote sensing analysis of Belmira's páramo vegetation with Landsat imagery". DYNA, 79/171, pp. 222-231.

Farley, F. y Kelly, F. (2004): "Effects of afforestation of páramo grassland on soil nutrient status". Forest Ecology and Management, 195, pp. 281-290.

Farley, K. (2007): "Grasslands to tree plantations: Forest transition in the Andes of Ecuador". Annals of the Association of American Geographers, 97/4, pp. 755-771.

Farley, K. (2008): "Plantaciones forestales y producción de servicios ambientales". Páramo, 26, pp. 3-25.

Ferweda, W. (2001): "La importancia global de los ecosistemas altoandinos y la visión de los donantes europeos sobre su conservación". Páramo, 13, pp. 7-9.

Flores, A. y Parión, H. (2005): "El turismo en Oyacachi: mucho más que aguas termales y paisaje". Páramo, 218, pp. 36-50.

Francou, B. y Vincent, C. (2007): Les glaciers à l'épreuve du climat. París, Belin, 247 pp.

Girard, S. (2005): "Les páramos, espace stratégique pour la gestion de l'eau dans les Andes septentrionales: le bassin-versant du río Ambato (Équateur)". Mappemonde, 78 , pp. 1-12

Graham, A. (2009): "The Andes: A geological overview from a biological perspective". Annals of the Missouri Botanical Garden, 96/3, pp. 371-385.

Guhl, E. (1982): Los páramos circundantes de la Sabana de Bogotá. Bogotá, Jardín botánico "José Celestino Mutis", 127 pp.

Harden, C. (2006): "Human impacts on headwater fluvial systems in the northern and central Andes". Geomorphology, 79, pp. 249-263.

Hedberg, I. y Hedberg, O. (1979): "Tropical-alpine lifeforms of vascular plants". Oikos, 33, pp. 297-307.

Henry, A.; Mabit, L.; Jaramillo, R. E.; Cartagena, Y. y Lynch, J.P. (2012): "Land use effects on erosion and carbon storage of the Río Chimbo watershed, Eduador". Plant Soil, pp. 1-15. 
Hofstede, R. (1995): "The effects of grazing and burning on soil and plant nutrient concentrations in Colombian páramo grasslands". Plant and Soil, 173, pp. 111-132.

Hofstede, R. (1999): "El Páramo como espacio para la fijación de carbono atmosférico". Páramo, 1, pp. 7-10.

Hofstede, R. y Aguirre, N. (1999): "Biomasa y dinámica del carbono en relación con las actividades forestales en la Sierra del Ecuador". Páramo, 1, pp. 31-46.

Hofstede, R.; Chilito, P. y Sandoval, E. (1995): "Vegetative structure, microclimate, and leaf growth of a paramo tussock grass species, in undisturbed, burned and grazed conditions". Vegetatio, 119, pp. 53-65.

Hofstede, R.; Groenendijk, J. P.; Coppus, R.; Fehse, J. C. y Sevink, J. (2002): "Impact of pine plantations on soils and vegetation in the Ecuadorian high Andes". Mountain Research and Development, 22, pp. 159-167.

Hofstede, R. y Mújica, E. (2002): "Birth of the Páramo Group: An international network of people, institutions, and projects working on páramo". Mountain Research and Development, 22, 1, pp. 83-84

Hofstede, R.; Segarra, P. y Mena, P. (eds.) (2003): Los páramos del mundo. Proyecto Atlas mundial de los páramos. Quito, Global peatland initiative/NC-UICN/EcoCiencia, 299 pp.

Josse, C; Cuesta, F; Navarro, G.; Barrena, V.; Cabrera, E. y Chacón-Moreno, E. et al. (2009): Ecosistemas de los Andes del norte y centro. Bolivia, Colombia, Ecuador, Perú y Venezuela. Lima, Secretaría General de la Comunidad Andina, 96 pp.

Kauffman, C. y Echavarría, M. (2012): "The evolution of water trust funds in Ecuador". WIT Transactions on Ecology and Environment, 168, pp. 3-16.

Keating, P. (2008): "The floristic composition and biogeographical significance of a megadiverse páramo site in the Ecuadorian Andes". Journal of the Torrey Botanical Society, 135, pp. 554-570.

Luteyn, J. L. (1992): "Páramos: why study them? “ en H. Baslev y J. L. Luteyn (eds.) Páramo: an Andean ecosystem under human influence. Londres, Academic Press, pp. 1-14.

Maffei, M. (2012): "Performance of Hargreaves equation in the estimating of reference evapotranspiration (ETO) in a zone of Andean paramo in Trujillo state, Venezuela". Revista de la Facultad de Agronomía, 29, 3, pp. 378-394.

Medina, G. y Mena, P. (2001): "Los páramos en el Ecuador", en P. Mena; A. G. Mena y R. Hofstede (eds.): Los páramos del Ecuador: particularidades, problemas y perspectivas. Quito, Abya Yala. Quito, pp. 1-23.

Mena, P. (2004): "Un resumen de la consultoría de políticas a escala regional del proyecto Páramo Andino". Páramo, 17, pp. 7-11.

Morales, J. A. y Estévez, J. V. (2006): “El páramo: ¿ecosistema en vía de extinción?”. Luna Azul, 22, pp. 1-13.

Nidia, C. A. y Cleef, A. M. (2009): "The páramo vegetation of Ramal de Guaracamal, Trujillo State, Venezuela. 2. Azonal vegetation”. Phytocoenologia, pp. 389-409. 
Podwojewski, P. y Poulenard, J. (2000): "Los suelos de los páramos del Ecuador". Páramo, 5, pp. 5-26.

Podwojewski, P.; Poulenard, J.; Zambrana, T. y Hofstede, R. (2002): "Overgrazing effects on vegetation cover and properties of volcanic ash soil in the páramo of Llangahua and La Esperanza (Tungurahua, Ecuador)". Soil Use and Management, 18, pp. 45-55.

Poulenard, J.; Podwojewski, P. y Herbillon, A. J. (2003): "Characteristics of non-allophanic Andisols with hydric properties from the Ecuadorian páramos". Geoderma, 117 , pp. 267-281.

Rabatel, A.; Francou, B.; Soruco, A.; Gómez, J.; Cáceres, B.; Ceballos, J. L.; Basantes, R.; Vuille, M.; Sicart, J. E.; Huggel, C.; Scheel, M.; Lejeune, Y.; Arnaud, Y.; Collet, M.; Condom, T.; Consoli, G.; Favier, V.; Jomelli, V.; Galárraga, R.; Ginot, P.; Maisincho, L.; Mendoza, J.; Ménégoz, M.; Ramírez, E.; Ribstein, P.; Suárez, W.; Villacís, M. y Wagnon, P. (2013): "Current state of glaciers in the tropical Andes: a multi-century perspective on glacier evolution and climate change". The Cryosphere, 7, pp. 81-102.

Ramón, G. (2002): "Visiones, usos e intervenciones en los páramos del Ecuador". Páramo, 12, pp. 47-54.

Rangel, O. (2000): Colombia Diversidad Biótica III. Bogotá, Instituto de Ciencias Naturales. Universidad Nacional de Colombia.

Rodríguez, F. y Behling, H. (2012): "Late quaternary vegetation, climate and fire dynamics, and evidence of early to mid-Holocene Polylepis forest in the Jimbura región of the southernmost Ecuadorian Andes". Palaeogeography, palaeoclimatology, Palaeoecology, 350-352, pp. 247-257.

Sauer, W. (1957): El mapa geológico del Ecuador. Quito, Editorial Universitaria.

Sierra, R. (1999): Propuesta Preliminar de un Sistema de Clasificación de Vegetaciónpara el Ecuador Continental. Quito, INEFAN/GEF-BIRF y EcoCiencia, 193 pp.

Sklenár, P.; Kecerová, A.; Macek, P. y Macková, J. (2010): "Does plant height determine the freezing resistance in the páramo plants?". Austral Ecology, 35/8, pp. 929-934.

Solórzano, P. (2005): "El proyecto de alpacas Alpa Huasy". Páramo, 18, pp. 23-35.

Troll, C. (1968): "The cordilleras of the tropical Americas: aspects of climatic, phytogeographical and agrarian ecology" en TROLL, C. (ed). Geoecology of the Mountainous Regions of the Tropical Americas. Bonn, UNESCO, pp. 15-56.

Van der Hammen, Th. (1997): "Ecosistemas terrestres: páramo", en Chaves, M. E. y Aarango, N. (eds). Informe nacional sobre el estado de la biodiversidad. Bogotá, Instituto Humboldt, pp. 9-37.

Varela, L. F. (2008): "The north Andes: the high plateau, an anthropogenic ecosistem". Pirineos, 163, pp. 85-95.

Villota, A.; León-Yánez, S. y Behling, H. (2012): "Vegetation and environmental dynamics in the Páramo of Jimbura región in the southeastern Ecuadorian Andes during the late Quaternary". Journal of South American Earth Sciences, 40, pp. 85-93.

Walter, H. (1998): Vegetació i zones climàtiques del món. Barcelona, PPU, 394.

WWF (2001): Biodiversity vision for the Northern Ecoregional Complex. Santiago de Cali, WWF, 34 pp. 


\section{RESUMEN}

Se presentan las características generales del páramo andino, un ecosistema de gran interés social y territorial poco conocido entre los geógrafos españoles. La síntesis desarrollada constituye un estado de la cuestión actualizado y contrastado sobre los principales aspectos que definen y caracterizan este espacio. La perspectiva adoptada tiene un marcado carácter sistémico y focaliza en cuestiones de orden ambiental, por ser este aspecto el que mejor engloba la complejidad actual del páramo. Así, después de glosar el encuadre geográfico del páramo andino, se repasa de forma sumaria los aspectos que mejor describen el beneficio social de este ámbito, a saber los aprovechamientos hídricos, la ocupación del suelo y el uso que realizan las sociedades implicadas. El propósito principal de este artículo es dar a conocer a los geógrafos ibéricos un ámbito de trabajo de gran actualidad entre los geógrafos latinoamericanos.

Palabras Clave: páramo; Andes; Latinoamérica; gestión del agua; ecosistemas tropicales.

\section{Abstract}

The most important characteristics of the Andean páramo are presented, an ecosystem of social and territorial interest, little known among Spaniard geographers. The synthesis developed constitutes a state of the art, updated and contrasted of the main appearances that define and characterise this space. The adopted approach has a systemic character and focuses in environmental questions, for being those ones who better embraces the páramo's current complexity. After summarizing the geographical setting of the Andean páramo, we review the subjects that better describe its social profit, which includes hydrological uses, land cover and land use activities carried by locals. The main purpose of this article is to let know to the Iberian geographers a subject of great actuality among Latin American geographers.

KEY WORDS: páramo; Andes; Latin America; water management; tropical ecosystems.

\section{RÉSUMÉ}

Cet article expose les caractéristiques générales du páramo andin : un écosystème de grand intérêt social et territorial peu connu des géographes espagnols. Cet article constitue un état de l'art actualisée et comparé des principaux aspects qui définissent et caractérisent cet espace. La perspective adoptée a un caractère systémique, et focalise sur les questions environnementales, qui sont au centre des enjeux actuels du páramo. Ainsi, après un résumé du cadre géographique du páramo andin, on détaille les aspects qui décrivent le mieux les bénéfices de ce milieu, à savoir les exploitations hydrauliques, l'occupation du sol et l'usage qu'en ont les sociétés locales. Lobjectif de ce travail est de faire connaître aux géographes ibériques un sujet de grande actualité entre géographes latino-américains.

MoTS ClÉs: páramo; Andes; Amérique latine; management de l'eau; écosystèmes tropicaux. 